\title{
Surface Modification of Fluorine-containing Polymers by Atmospheric Pressure Plasma Jet with Negative Substrate Biasing
}

\author{
Masaaki Nagatsu ${ }^{1,2,3^{*}}$, Masahiro Kimpara ${ }^{2}$, Rui $\mathrm{Hu}^{3,4}$, and Tomy Abuzairi ${ }^{5}$ \\ ${ }^{1}$ Research Institute of Electronics, Shizuoka University, \\ ${ }^{2}$ Graduate School of Integrated Science and Technology, Shizuoka University, \\ ${ }^{3}$ Graduate School of Science and Technology, Shizuoka University, \\ 3-5-1 Johoku, Naka-ku, Hamamatsu, 432-8561, Japan \\ ${ }^{4}$ Institute of Plasma Physics, Chinese Academy of Sciences, Hefei 230031, P.R. China \\ ${ }^{5}$ Department of Electrical Engineering, University of Indonesia, Depok 16424, Indonesia \\ *nagatsu.masaaki@shizuoka.ac.jp
}

Fluoropolymers are difficult materials to modify the surfaces because of strong C-F bonds. In this study, the surface modification of fluoropolymer films such as polytetrafluoroethylene (PTFE) with amino groups was performed by using $\mathrm{He} / \mathrm{NH}_{3}$ gas mixture atmospheric pressure plasma jets with negatively-biased substrate. It is expected that the ion bombardment effect onto the polymer surface due to negative substrate bias will make dangling bonds over the polymer surface and eventually promote the surface modification. From XPS analysis, we confirmed the breaking C-F bonds and creating $\mathrm{C}-\mathrm{C}, \mathrm{C}=\mathrm{O}$ or $\mathrm{C}-\mathrm{N}$ bonds in $\mathrm{C} 1 \mathrm{~s}$ spectrum and increasing nitrogen-containing functional groups in $\mathrm{N}$ 1s spectrum. Fluorescence patterns were observed on the amino group functionalized PTFE surface by using the fluorescent dyes selectively connecting with the amino groups. With a spectrophotometric method, the surface concentration of amino groups introduced onto the PTFE surface was evaluated to be roughly $3.86 \mathrm{nmol} / \mathrm{cm}^{2}$.

Keywords: Surface modification, Fluoropolymers, Polytetrafluoroethylene, Atmospheric pressure plasma jet, Amino group

\section{Introduction}

Fluoropolymers, such as polytetrafluoroethylene (PTFE), perfluoroalkoxy polymer (PFA), fluorinated ethylene-propylene (FEP), etc., show their excellent dielectric, thermal and mechanical properties, in addition to high chemical stability. However, their poor adhesion properties with other materials are strong drawbacks for their uses in various industrial fields. Therefore, proper surface treatments are crucial for any applications to use them for adhesion with other materials. So far, many research have been carried out to improve the surface adhesion property of fluoropolymers by making use of wet chemical approaches with sodium naphthalenide treatment [1-3], photochemical modification with hydrazine [4], ion beam irradiation [5] or surface modification using low pressure plasmas [6-12] and atmospheric pressure plasmas [13-15]. Recently, novel approach with a heat-assisted plasma surface modification at atmospheric pressure has been reported to demonstrate the improved adhesion between PTFE and rubbers [16]. However, PTFE surface was heated up to about $250^{\circ} \mathrm{C}$ by plasma irradiation and external heater, which is close to the maximum operating temperature of PTFE at $260^{\circ} \mathrm{C}$. Indeed, PTFE surface might be locally heated by surface recombination of plasma species via plasma-surface interaction, so temperature of the outmost surface area would become near or higher than melting point of PTFE.

In this study, therefore, we propose lowtemperature surface modification technique of PTFE by means of environmentally-friendly dry plasma processing under atmospheric pressure. The surface modification of PTFE sheets with 
amino groups was performed by using a $\mathrm{He} / \mathrm{NH}_{3}$ gas mixture atmospheric pressure plasma jet(APPJ) with negatively biased substrate. Here, we expect that $\mathrm{C}-\mathrm{F}$ and $\mathrm{C}-\mathrm{C}$ bonds of PTFE surface will be broken by the ion bombardment due to negative substrate bias and eventually amino group modification can be enhanced via interaction with $\mathrm{NH}$ or $\mathrm{NH}_{2}$ radicals. Here we examine the chemical bonds on the surface of plasma treated PTFE samples by making use of X-ray photoelectron spectroscopy (XPS). We also study the pattering of amino group modification onto the PTFE surface by using the fluorescent dyes selectively connecting with the amino groups. With a spectrophotometric method [17], the surface concentration of amino groups introduced onto the PTFE surface is estimated.

\section{Experimental}

The experimental setup of the APPJ with a single electrode configuration is shown in Fig. 1. A glass tube having $6 \mathrm{~mm}$ in outer diameter and $4 \mathrm{~mm}$ in inner diameter is wrapped by a copper tape electrode about $60 \mathrm{~mm}$ from the exit tip of the tapered glass tube with an aperture diameter of 700 $\mu \mathrm{m}[18]$. The discharge is produced in a glass tube with about $700 \mathrm{sccm} \mathrm{He}$ gas flow and $10 \mathrm{sccm} \mathrm{NH}$ gas flow, applying high voltage square-wave pulses of $\pm 7.5 \mathrm{kV}$ amplitude, $5 \mathrm{kHz}$ frequency, and $50 \%$ duty ratio obtained by employing a multifunction generator (NF, WF1948) and AC/DC amplifier (NF, HVA4321). To control the gas flow rate is critical to obtain a stable plasma discharge in the present APPJ device without any damage of capillary tip. The value of gas flow used in this experiment $(\sim 700$ $\mathrm{sccm}$ ) was determined from the stable discharge condition of atmospheric pressure plasma jet at appropriate applied voltages [19-21]. Currentvoltage waveforms of discharges are measured using a digital oscilloscope (Tektronix, DPO 4104B-L) connected to a high voltage probe (Tektronix, P6015A), and a current probe (Pearson, 4100) on power electrode line. The net plasma bullets ejecting from the exit tip were detected by means of a metal probe set inside a grounded shield box, as shown in Fig. 1 [18].

In the present experiment, we used PTFE films with a thickness of $1 \mathrm{~mm}$, which are available from Nilaco Corp. They were fixed on the stage by a carbon tape and masked by a $\mathrm{Cu}$ grid with an aperture hole size of $100 \mu \mathrm{m}$, as illustrated in Fig. 2. The visualization of the plasma surface modification was done by the chemical derivatization method using specific fluorescence dyes and fluorescence microscopy. Here, we used the fluorescent dye Alexa Fluor ${ }^{\circledR} 488$ sulfodichloro-phenol (5-SDP) ester (Life Technologies) which reacts with the amine moieties patterned on the surface of the polymer, and emits

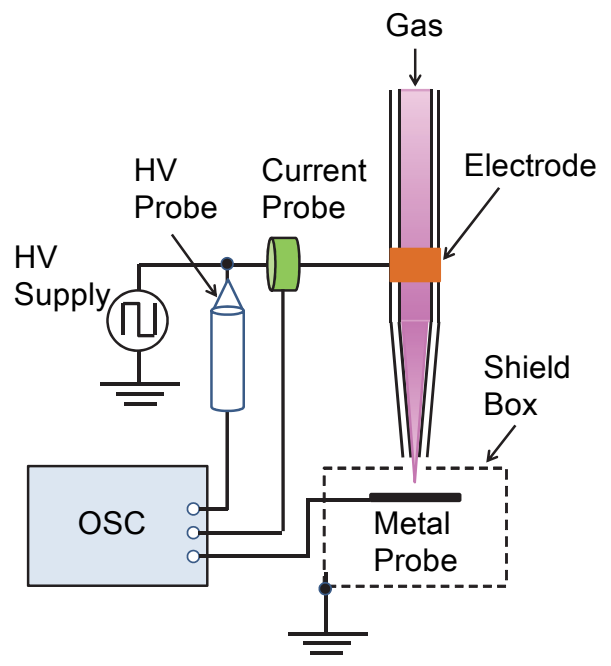

Fig. 1. Schematic drawing of the experimental setup for measurement of current-voltage characteristics.

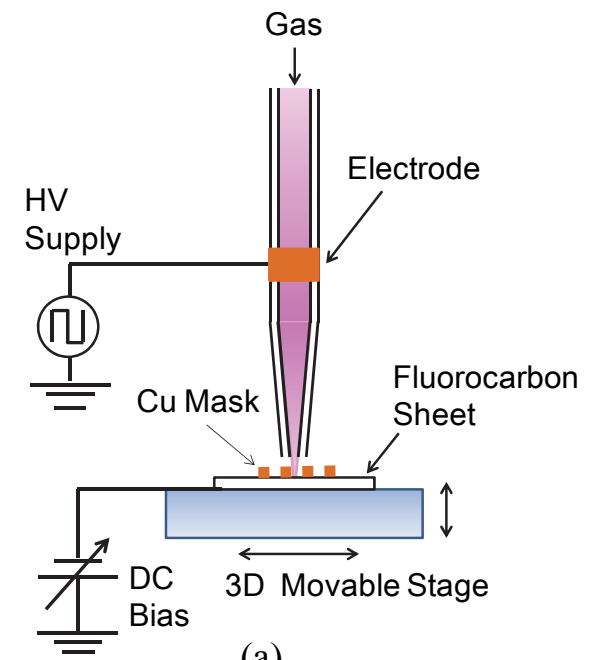

(a)

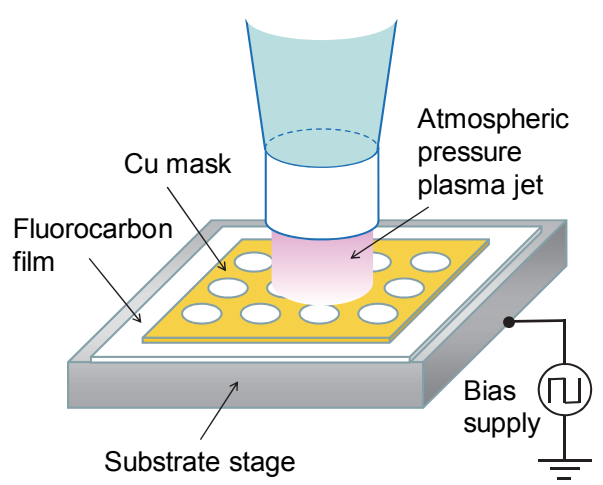

(b)

Fig. 2. Schematic drawings of (a) proposed surface modification method by APPJs with negative substrate bias and (b) PTFE sample with $\mathrm{Cu}$ grid mask. 
green fluorescence at $519 \mathrm{~nm}$ when excited by 495 $\mathrm{nm}$ light. The images of the functionalized areas were measured by fluorescence microscope (Leica, DMI 4000). Samples were also analyzed by an XPS(Shimadzu, Axis Ultra DLD).

\section{Results and discussion}

\subsection{Current-voltage characteristics}

A typical current-voltage characteristic of the APPJ discharge in the experimental setup given in Fig. 1 is shown in Fig. 3, where applied voltage and current waveforms are measured simultaneously. Both the rise and fall times of the applied high voltage pulses are about $25 \mu \mathrm{s}$. It is seen that during the rising and falling phases of voltage waveform, fishbone like dielectric barrier discharge currents are observed. Furthermore, using the metal probe, we observed spiky single current pulses during the rising phase, where these spiky currents contribute to the surface modification of PTFE sample.

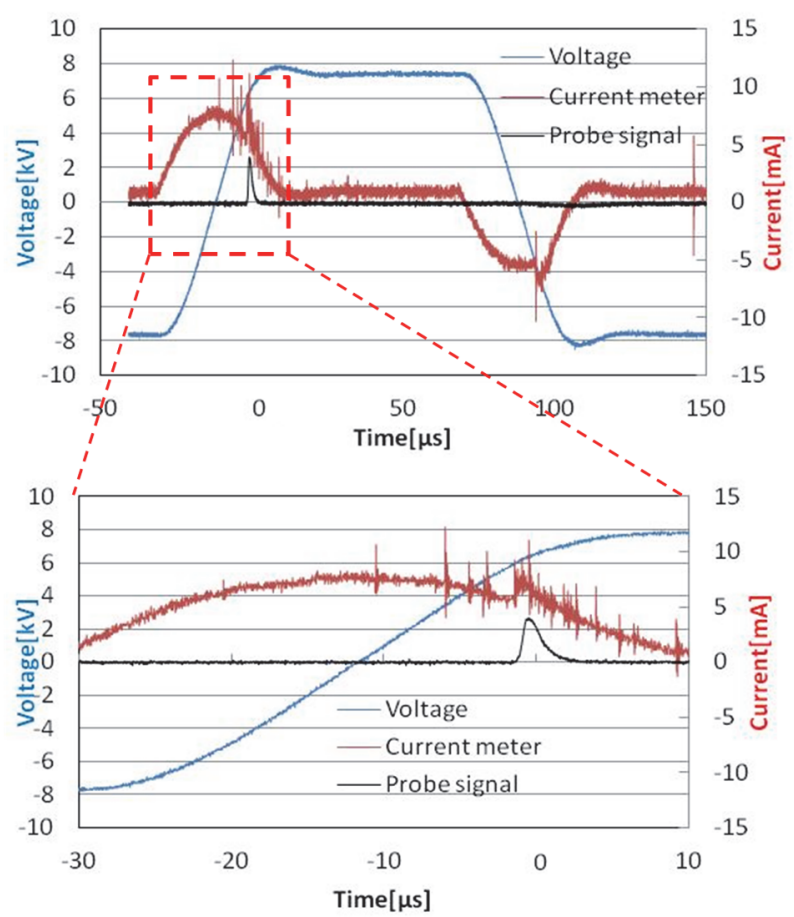

Fig. 3. Typical waveforms of applied voltage, current and probe signal.

\subsection{Fluorescent patterning of amino-group} modified PTFE

To confirm the effect of negative substrate bias on the amino group modification onto the PTFE surface treated by the $\mathrm{He} / \mathrm{NH}_{3}$ APPJs, we have tested the chemical derivatization using fluorescent dye, Alexa Fluor ${ }^{\circledR} 488$ 5-SDP. As the first step, the PTFE samples covered with a $\mathrm{Cu}$ grid mask were treated by pure He APPJ for $20 \mathrm{~s}$ under different conditions of substrate bias voltages from 0 to $-500 \mathrm{~V}$. Then, samples were treated by $\mathrm{He} / \mathrm{NH}_{3}$ APPJs for $160 \mathrm{~s}$ without substrate bias. Figure 4 shows the bright-field image of $\mathrm{Cu}$ mask and fluorescent images of PTFE samples treated by $20 \mathrm{~s}$ He APPJ irradiation with different bias voltages of $0,-200,-300,-400$ and $-500 \mathrm{~V}$ as pretreatment and successively treated by $160 \mathrm{~s} H \mathrm{He} / \mathrm{NH}_{3}$ APPJ irradiation without bias as post-treatment. With an increase of the bias voltage during $20 \mathrm{~s}$ pretreatment, it is seen that fluorescent image became brighter and brighter, which might be attributed to an increase of amino group population introduced on the surface of PTFEs.
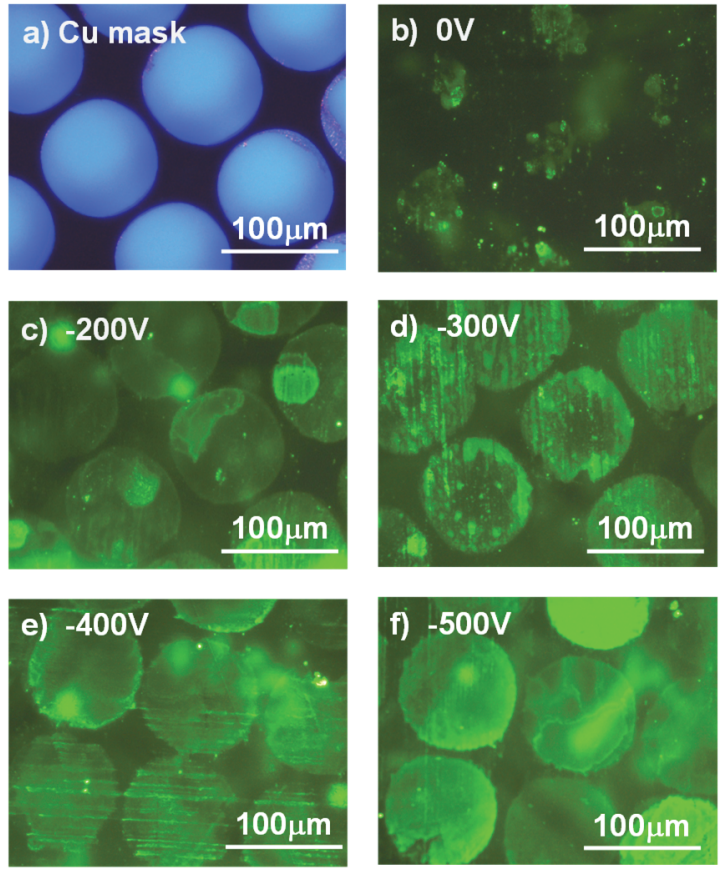

Fig. 4. (a) Bright-field image of $\mathrm{Cu}$ mask and fluorescent images of PTFE samples treated by $20 \mathrm{~s}$ He APPJ irradiation with different bias voltages of (b) $0 \mathrm{~V}$, (c) -200 $\mathrm{V}$, (d)-300 V, (e) $-400 \mathrm{~V}$, and (f) $-500 \mathrm{~V}$, and successively treated by $160 \mathrm{~s} \mathrm{He} / \mathrm{NH}_{3} \mathrm{APPJ}$ without bias.

\subsection{XPS analysis of plasma modified PTFE}

To investigate the chemical bonds of PTFE surfaces, we performed the XPS analysis of PTFE samples. First, we tested XPS spectra of PTFE surface treated by pure He APPJs for 3 min with and without negative DC bias voltage at $-500 \mathrm{~V}$. Figure 5 shows the XPS spectra of C $1 \mathrm{~s}, \mathrm{~N} 1 \mathrm{~s}$ and $\mathrm{O} 1 \mathrm{~s}$ of untreated PTFE sample and He APPJ with bias at $500 \mathrm{~V}$ and without bias. All the XPS spectra were calibrated at $292.0 \mathrm{eV}$ of $\mathrm{CF}_{2}$ bond beforehand to cancel the chemical shift due to neutralization procedure of accumulated charge. Even in the case of He APPJ irradiation without bias, we 
observed small peaks at $\sim 401 \mathrm{eV}(\mathrm{N}-\mathrm{C}-\mathrm{O})$ and $\sim 402.5 \mathrm{eV}\left(\mathrm{NH}_{3}{ }^{+}\right)$in $\mathrm{N} 1 \mathrm{~s}$ and $\sim 532(\mathrm{C}=\mathrm{O}), \sim 533$ (C-O), and $\sim 534.5 \mathrm{eV}(\mathrm{O}-\mathrm{C}=\mathrm{O})$ in $\mathrm{O} 1 \mathrm{~s}$ spectra, as shown in Figs. 5(b) and (c). These components are possibly originated from active $\mathrm{O}$ and $\mathrm{N}$ atomic components generated in air region below the exit tip of He APPJs.

(a)

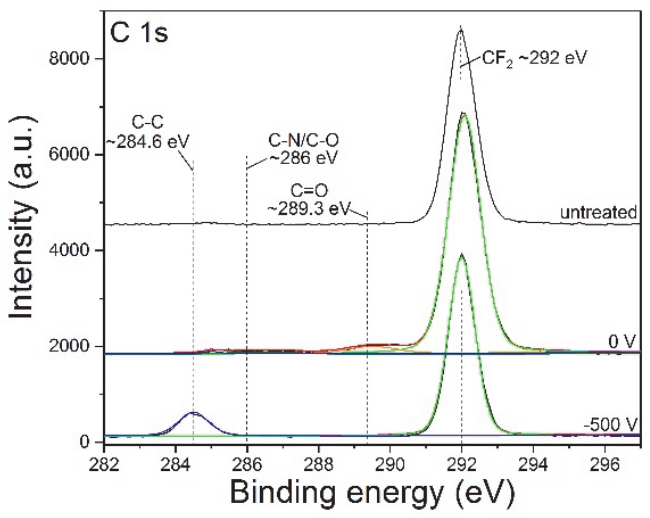

(b)

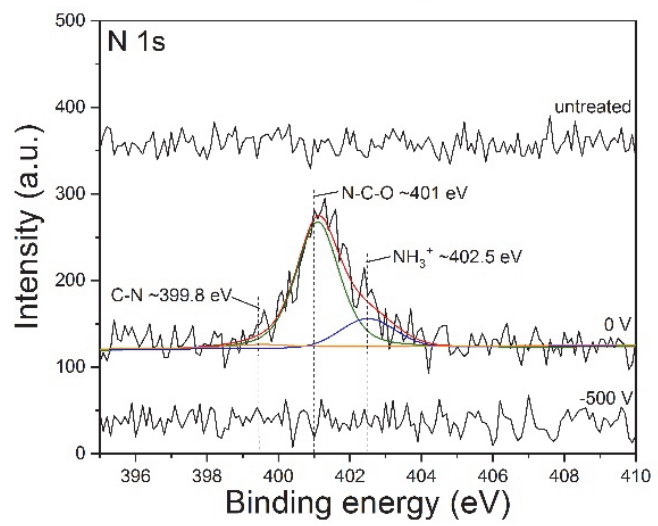

(c)

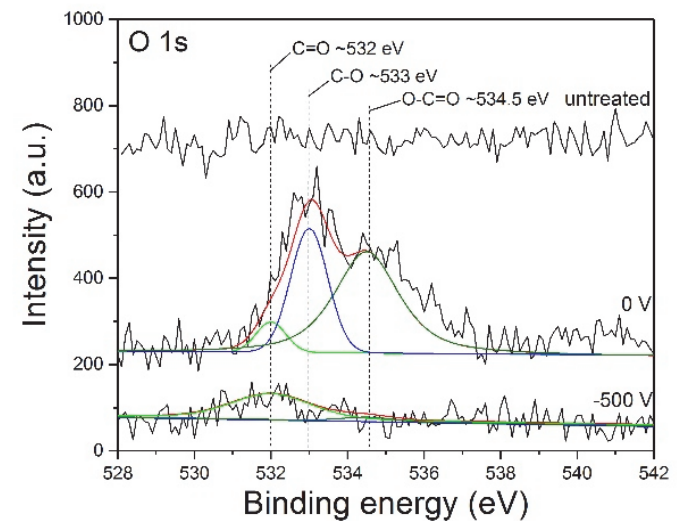

Fig. 5. XPS spectra of (a) C 1s, (b) N 1s and (c) O 1s of untreated PTFE sample and pure He APPJ with bias and without DC bias at $-500 \mathrm{~V}$, respectively.

In the case of He APPJ with bias at $-500 \mathrm{~V}, \mathrm{C} 1 \mathrm{~s}$ spectrum shows a small peak at $284.6 \mathrm{eV}(\mathrm{C}-\mathrm{C})$, however, $\mathrm{N} 1 \mathrm{~s}$ and $\mathrm{O} 1 \mathrm{~s}$ spectra indicate negligible small changes. These results may suggest that the negative bias of substrate stage causes the stronger bombarding effect of APPJs on the PTFE surface, so that the surface modification was damaged by ion bombardment.

Next, we investigated the effect of addition of $\mathrm{NH}_{3}$ gas into He carrier gas on the PTFE surface treated by $\mathrm{He} / \mathrm{NH}_{3}$ APPJs for different bias voltages during a fixed period of $3 \mathrm{~min}$. In these experiments, negative DC bias voltages applied at the substrate stage was varied from 0 to $-800 \mathrm{~V}$ during $3 \mathrm{~min}$ irradiation period.

(a)

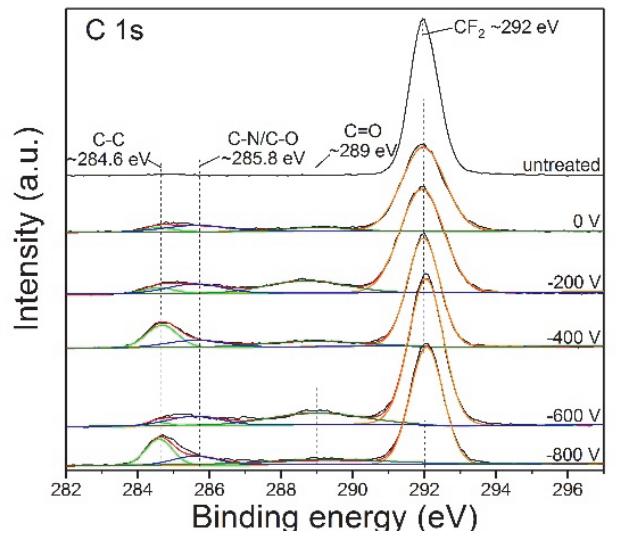

(b)

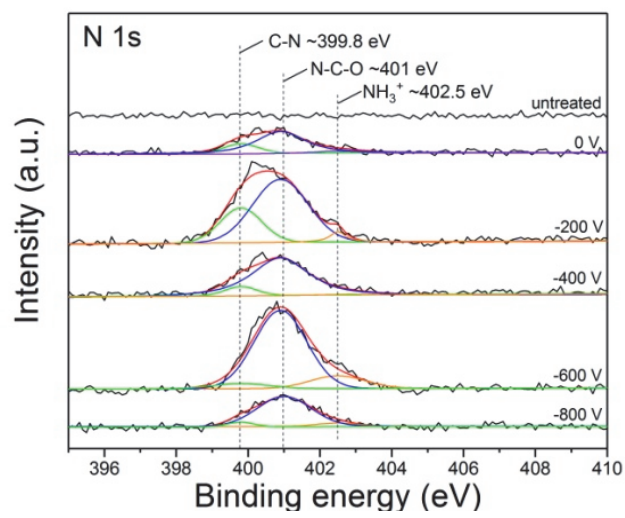

(c)

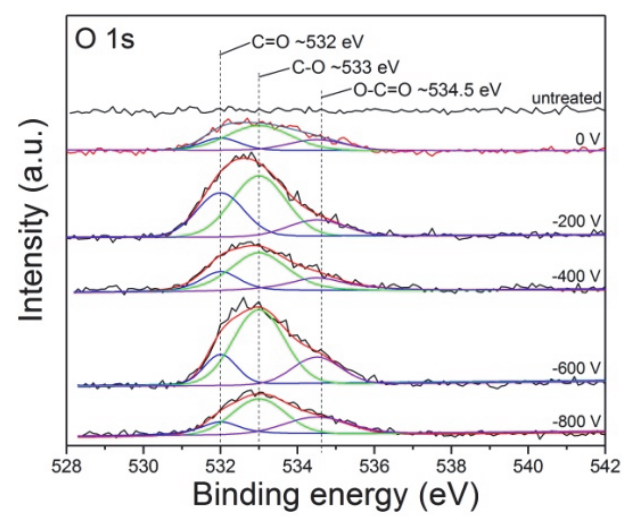

Fig. 6. XPS spectra of (a) $\mathrm{C} 1 \mathrm{~s}$, (b) $\mathrm{N} 1 \mathrm{~s}$, and (c) $\mathrm{O} 1 \mathrm{~s}$ for $\mathrm{H} / \mathrm{NH}_{3}$ plasma treated PTFE films for $3 \mathrm{~min}$ with different negative bias voltages from 0 to $-800 \mathrm{~V}$.

Figure 6 shows the XPS spectra of C 1s, N 1s and $\mathrm{O} 1 \mathrm{~s}$ of various PTFE samples treated by $\mathrm{He} / \mathrm{NH}_{3}$ APPJs with different DC bias voltages. Different from the results of pure He APPJ irradiation, $\mathrm{N} 1 \mathrm{~s}$ signal clearly appeared and was enhanced even in the case of $\mathrm{He} / \mathrm{NH}_{3} \mathrm{APPJ}$ without bias. It means 
the $\mathrm{NH}_{3}$ gas addition is effective for introducing nitrogen-containing groups onto the PTFE surface. With an increase of negative bias voltages, $\mathrm{CF}_{2}$ peak intensity dropped and C-C, C-N/C-O (285.8 eV), or $\mathrm{C}=\mathrm{O}(289 \mathrm{eV})$ peaks are appeared. As shown in Figs. 6(b) and (c), deconvoluted $\mathrm{N} \mathrm{1s}$ and $\mathrm{O} 1 \mathrm{~s}$ spectra show analogous spectra to those in Figs. 5 (b) and (c). With an increase of bias voltage, Fig. 6 shows repetitive behaviors, that is, the similar spectra were observed at $-200 \mathrm{~V}$ and $-600 \mathrm{~V}$, and also at $-400 \mathrm{~V}$ and $-800 \mathrm{~V}$. It might be related with the continuous etching by hydrogen atoms of the outmost surface layer of PTFE. To clarify the behaviors in detail, it might be rather important to study the XPS spectra at different treatment times under the fixed bias voltages.

3.4 Estimation of surface concentration of amino groups on PTFE sheet

The amino group population of the plasmatreated PTFE samples was analyzed by a conventional spectrophotometric determination method using sulfo-LC-SPDP according to the specific chemical procedure [17], as illustrated in Fig. 7. Amino-functionalized PTFE samples were dispersed into $200 \mu \mathrm{L}$ of $10 \mathrm{mM}$ sulfo-LC-SPDP in phosphate buffer saline (PBS) and reacted for 30 min under a dark condition with an ultrasonication of each $5 \mathrm{~min}$.

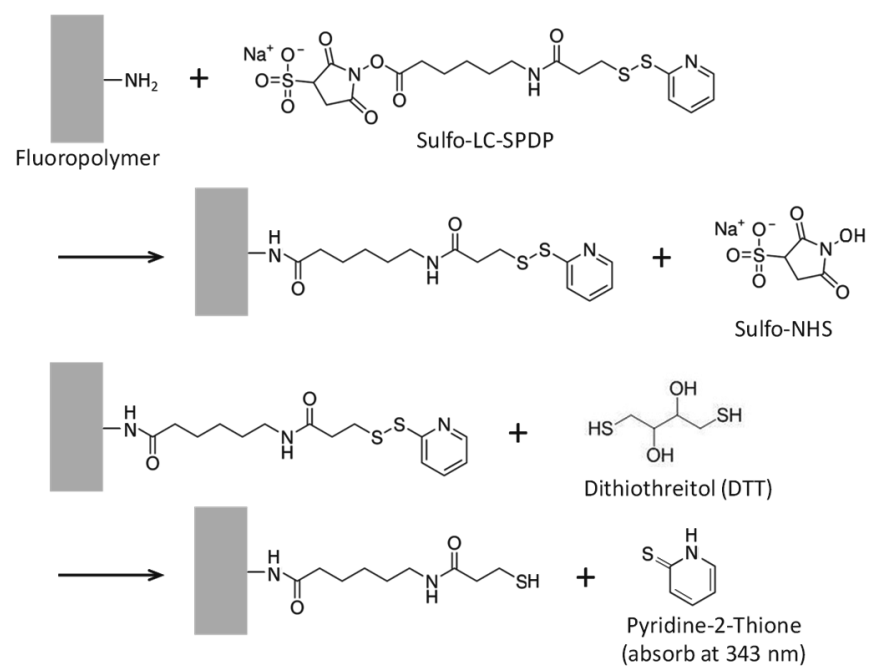

Fig. 7. Illustration of spectrophotometric determination of amino groups on the PTFE samples.

Then sulfo-LC- SPDP reacted PTFE samples were rinsed with PBS solution thoroughly. After this process, PTFEs with sulfo-LC-SPDP complexes were reacted with $300 \mu \mathrm{L}$ of $20 \mathrm{mM}$ dithiothreitol in PBS solution under dark condition. After a $15 \mathrm{~min}$ reaction, the PTFE samples were separated and the cleaved product (i.e., pyridine-2-thione) liberated from the sulfo-LC-SPDP in the supernatant liquid was determined by measuring the absorbance at 343 nm using a microplate reader (Infinite 200 Pro, TECAN). Figure 8 shows the UV-Vis absorption spectrum of supernatant liquid. At $343 \mathrm{~nm}$, we can see a signal of pyridine- 2 thione, which was magnified and plotted inside Fig. 8.

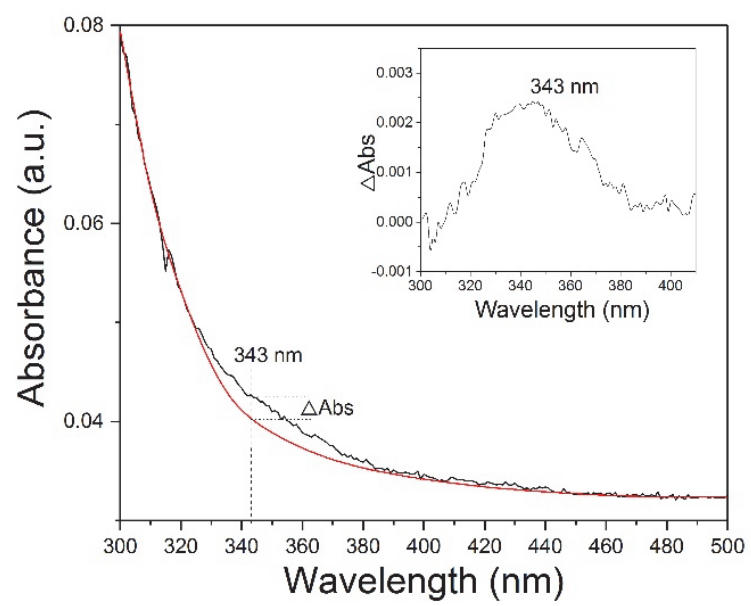

Fig. 8. UV-Vis absorption spectrum of pyridine-2-thione in supernatant liquid.

The number of amino groups in PTFE samples was quantitatively determined from the calibration curve using the extinction coefficient of pyridine-2thione at $343 \mathrm{~nm}$ (i.e., $8.08 \times 10^{3} \mathrm{M}^{-1} \mathrm{~cm}^{-1}$ ). Taking $\triangle \mathrm{Abs} \sim 0.0024$ from Fig. 8 , we calculated surface density of amino groups introduced onto the surface of PTFE samples by dividing by treated surface area, then we obtained $3.86 \mathrm{nmol} / \mathrm{cm}^{2}$ or $23.2 / \mathrm{nm}^{2}$. From this value, we can simply calculate an average occupation area per amino group to be $0.043 \mathrm{~nm}^{2}$. Assuming a circle for each amino group location simply, we obtain $0.117 \mathrm{~nm}$ as its radius. This length is smaller than the end-to-end length of $\mathrm{N}-\mathrm{H}$ bond, about $0.2 \mathrm{~nm}$. Therefore, it is considered that measured surface concentration of amino groups is corresponding to almost full occupation of amino groups over the PTFE surface.

Lastly, we have carried out the measurement of water contact angle using purified water. Here we treated PTFE surface by $3 \mathrm{~min} \mathrm{He} / \mathrm{NH}_{3}$ APPJ under a constant dc bias at $-200 \mathrm{~V}$. Figure 9 shows the photos taken for untreated PTFE sample and plasma-treated PTFE samples, respectively. As found from Fig. 9, initial water contact angle is evaluated to be about $118^{\circ}$, while it dropped down to about $49^{\circ}$ after $3 \mathrm{~min}$ plasma treatment. The change of surface wettability from hydrophobic to hydrophilic is attributed to the surface modification with amino groups. 


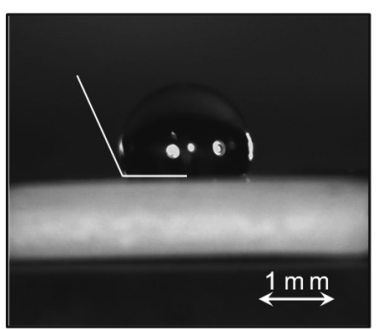

(a)

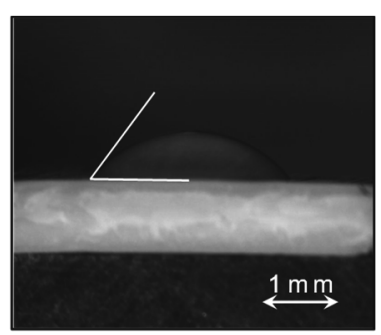

(b)
Fig. 9. Photos of water contact angle for the cases of (a) untreated and (b) $\mathrm{He} / \mathrm{NH}_{3}$ APPJ treated PTFE for $3 \mathrm{~min}$ under the condition of DC bias of $-200 \mathrm{~V}$.

\section{Conclusion}

In this study, the surface modification of PTFE films with amino groups was performed by using $\mathrm{He} / \mathrm{NH}_{3}$ gas mixture atmospheric pressure plasma jets with negatively-biased substrate. From XPS analysis, we confirmed the breaking $\mathrm{C}-\mathrm{F}$ bonds and creating $\mathrm{C}-\mathrm{C}, \mathrm{C}=\mathrm{O}$ or $\mathrm{C}-\mathrm{N}$ bonds in $\mathrm{C} 1 \mathrm{~s}$ spectrum and increasing nitrogen-containing functional groups in $\mathrm{N}$ 1s spectrum. Fluorescence patterns were clearly observed on the amino group functionalized PTFE surface by using the fluorescent dyes. Using a spectrophotometric method with Sulfo-LC-SPDP and DTT reaction, the surface concentration of amino groups introduced onto the PTFE surface was estimated as roughly $3.86 \mathrm{nmol} / \mathrm{cm}^{2}$. From the water contact angle measurement, we confirmed that water contact angle of untreated PTFE sample was drastically decreased from $118^{\circ}$ down to $49^{\circ}$ after $3 \mathrm{~min}$ $\mathrm{He} / \mathrm{NH}_{3}$ APPJ treatment under the condition of $\mathrm{dc}$ bias of $-200 \mathrm{~V}$. From the present study, we can conclude that PTFE surface was almost fully functionalized with amino groups over the PTFE surface at low temperature by using $\mathrm{He} / \mathrm{NH}_{3} \mathrm{APPJ}$ under the negative substrate bias condition.

\section{Acknowledgement}

This work was partly supported by Grants-inAid for Scientific Research (A) (No. 25246029) and Scientific Research (B) (No. 17H02804) from the Japan Society for the Promotion of Science.

\section{References}

1. K. Ha, S. McClain, S. L. Suib, and A. Garton, J.
Adhes., 33 (1991) 169.

2. L. M. Siperko and R. R. Thomas, J. Adhes. Sci. Technol., 3 (1989) 157.

3. M. Gabriel, K. Niederer, M. Becker, C. M. Raynaud, C-F. Vahl, and H. Frey, Bioconjugate Chem., 27 (2016) 1216.

4. U. Meyer, S. Kostler, V. Ribitsch, and W. Kern, Macromol. Chem. Phys. 206 (2005) 210.

5. S. W. Lee, J. W. Hong, M. Y. Wye, J. H. Kim, H. J. Kang, and Y. S. Lee, Nucl. Instr. Meth. B, 219-220 (2004) 963.

6. E. T. Kang, K. L. Tan, K. Kato, Y. Uyama, and Y. Ikada, Macromolecules, 29 (1996) 6872.

7. J. P. badey, E. Espuche, D. Sage, B. Chabert, Y. Jugnet, C. Batier, and T. M. Duc, Polymer, 17 (1996) 1377.

8. D. J. Wilson, R. L. Williams, and R. C. Pond, Surf. Interface Anal., 31 (2001) 385.

9. T. K. Markkula, J. A. Hunt, F. R. Pu, and R. L. Williams, Surf. Interface Anal., 34 (2002) 583.

10. N. Vandencasteele, D. Merche, and F. Reniers, Surf. Interface Anal., 38 (2006) 526.

11. T-K. Lin, S-J. Wu, C-K. Peng, and C-H. Yeh, Polym. Int., 58 (2009) 46.

12. W. Hai, T. Hi, K. Shimizu, and T. Yajima, J. Photopolym. Sci. Technol., 28 (2015) 479.

13. C. Z. Liu, J. Q. Wu, L. Q. Ren, J. Tong, J. Q. Li, N. Cui, N. M. D. Brown, and B. J. Meenan, Mater. Chem. Phys., 85 (2004) 340.

14. Z. Fang, L. Hao, H. Yang, X. Xie, Y. Qiu, and K. Edmund, Appl. Surf. Sci., 255 (2009) 7279.

15. J. H. Noh, H. K. Baik, I. Noh, J-C. Park, and I-S. Lee, Surf. Coat. Technol., 201 (2007) 5097.

16. Y. Ohkubo, K. Ishihara, H. Sato, M. Shibahara, A. Nagatani, K. Honda, K. Endo, and Y. Yamamura, RSC Adv., 7 (2017) 6432.

17. T. T. Ngo, J. Biochem. Biophys. Methods, 12 (1986) 349.

18. T. Abuzairi, M. Okada, S. Bhattacharjee, and M. Nagatsu, Appl. Surf. Sci., 390 (2016) 489.

19. R. Kakei, A. Ogino, F. Iwata, and M. Nagatsu, Thin Solid Films, 518 (2010) 3457.

20. T. Abuzairi, M. Okada, Y. Mochizuki, N. R. Poespawati, R. W. Purnamaningsih, and M. Nagatsu, Carbon, 89 (2015) 208.

21. I. Motrescu and M. Nagatsu, ACS Appl. Mater. Interfaces, 8 (2016) 12528. 